\section{OTU-07 NEAR FOCUS NARROW AND IMAGING DRIVEN ARTIFICIAL INTELLIGENCE FOR THE DIAGNOSIS OF GASTRO-OESOPHAGEAL REFLUX DISEASE}

${ }^{1}$ Shraddha Gulati*, ${ }^{2}$ Julius Bernth, ${ }^{2} J$ unkai Liao, ${ }^{2}$ Dmytro Poliyivets, ${ }^{1}$ Sukhdev Chatu, ${ }^{1}$ Andrew Emmanuel, ${ }^{1}$ Amyn Haji, ${ }^{2}$ Hongbin Liu, ${ }^{1}$ Bu'Hussain Hayee. ${ }^{1}$ King's College

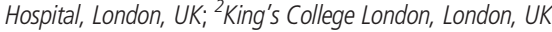

\subsection{6/gutjnl-2019-BSGAbstracts.7}

Introduction Gastro-oesophageal reflux disease(GORD) is a common condition carrying an arduous process for diagnosis. Symptom questionnaires and proton-pump inhibitor challenge lack reliability and white light endoscopy (WLE) is often normal. Objective measurement of oesophageal acid exposure time (AET) require invasive testing. Changes in intrapapillary capillary loops (IPCLs) identified using narrow band imaging (NBI) have been proposed as a marker for reflux. We evaluated a near focus (NF-NBI) driven artificial intelligence(AI) model for the diagnosis of GORD.

Methods Patients with symptoms of GORD (recorded using the Reflux Disease Questionnaire(RDQ)) were prospectively recruited over 10 months. Upper endoscopy recorded multiple NF-NBI images, video and biopsies of the lower oesophagus. If endoscopy using High-Definition WLE was normal, a $\mathrm{pH}$ recording capsule was placed. Patients were defined according to Lyon criteria; Erosive oesophagitis(EO);non-erosive reflux disease(NERD); functional heartburn(FH).

Two forms of AI were developed and evaluated to automate regions of interest (ROI) and detect IPCLs and morphology: computer vision (CV) and deep convoluted neural network(DCNN) using Resnet50. DCNN was evaluated using training: unseen testing dataset ratios of 50:50 (3872:4280 images) and 75:25 (6484:1668 images). For the purposes of training the AI models, EO and NERD cases were combined as 'GORD'. A novel combined classifier (CC) of both AI methods was evaluated.

Results 78 consecutive patients were recruited. n=68 (46 Female, 44.41 \pm 12.91 years): GORD $n=27$ (EO $n=6$, NERD $\mathrm{n}=21$ ) and $\mathrm{FH} \mathrm{n}=41$ were analysed. The mean IPCL per ROI count was greater in GORD vs FH: $33.2 \pm 5.19$ vs $28.1 \pm 5.42 \mathrm{p}=0.0003$ and was used as the primary diagnostic tool. IPCL morphology for GERD vs FH: length 16.29 vs 16.98, $\mathrm{p}=0.19$; width 7.8 vs $7.8, \mathrm{p}=0.98$; red 118.8 vs $120.6, \mathrm{p}=0.44$; green 114.3 vs 118 , p0.004; blue 94.24 vs $97.54 \mathrm{p}=0.07$

With CV: mean IPCLs/ROI (threshold 27.6) had sensitivity, specificity, AUC: 88.9, 58.5, $0.76(\mathrm{p}=0.0003)$ for GORD. With DCNN 50:50 these results were 58\%, 86\% and $76 \%$ respectively. DCNN 75:25 produced 67\%, 92\%, 83\% respectively.

CC improved overall specificity(89.1\%) and accuracy (78.1\%) but not sensitivity(63\%).

Conclusion AI using NF-NBI is a novel method for the diagnosis of GORD. With increased data, improvements in diagnostic accuracy is achieved further improved using a CC. This model has the potential to provide a reliable safe single-test diagnosis of GORD.

\section{Abstracts of Distinction}

\section{ATH-01 THE OVESCO OVER THE SCOPE CLIP (OTSC) REDUCES GASTROINTESTINAL HAEMORRHAGE RE-BLEEDING RATES-FIRST UK SERIES}

Timothy Keen*, Alvin Ochieng, Philip Boger, Thomas Hollingworth, Praful Patel, Imdadur Rahman. University Hospital Southampton, NHS Foundation Trust, Southampton, UK

\subsection{6/gutjnl-2019-BSGAbstracts.8}

Introduction The OVESCO over the scope clip (OTSC) has recently been described in treating upper gastrointestinal (UGI) haemorrhage in Europe. The aim of this study was to determine whether use of the OTSC was associated with lower UGI haemorrhage re-bleeding rates and mortality in the UK.

Methods Consecutive episodes of UGI haemorrhage treated with the OTSC were identified from a prospective database in a UK tertiary centre over a 2 -year period. Treatment with OTSC was opted for patients with high risk features or failed conventional therapy. Over the same time period, patients within the same centre with UGI haemorrhage treated with standard endoscopic therapy were retrospectively identified as a control group. Patient demographics, 7 day re-bleeding rate, 30 day re-bleeding rate and 30 day mortality rates were compared from each group. T-test and Pearson's Chi-square test were used as appropriate.

Results 408 episodes of UGI haemorrhage were identified requiring endoscopic intervention. Of these 59 high risk lesions were treated with the OTSC; forest $1 \mathrm{a}-20 \%, 1 \mathrm{~b}-31 \%$, $2 \mathrm{a}-29 \%, 2 \mathrm{~b}-20 \%$. The sites of lesions treated with the OTSC included oesophagus (10\%), stomach (20\%) and duodenum (70\%). Pathology included 48 (81\%) ulcers, 4 (6.8\%) Mallory-Weiss tears, 3 (5\%) Dieulafoy, 2 (3.4\%) post-angiographic coil ulcer, 1 (1.7\%) post-EMR and 1 (1.7\%) anastomotic bleed. 349 episodes were identified for the control group. Median age was 70 years in the OTSC group versus 72 years in the control group $(p=0.21)$. There was no significance difference in sex between the OTSC group and the control group (64\% male vs $70 \%$ male, $\mathrm{p}=0.41)$.

Compared to the control group, the OTSC group had lower 7 -day re-bleeding rate $(12 \%$ vs $3.4 \%, p=0.048)$ and a trend towards lower 30 -day re-bleeding rate $(15 \%$ vs $6.8 \%$, $\mathrm{p}=0.10)$. Although all-cause mortality in OTSC group was similar to the control group $(6.8 \%$ vs $8.3 \%, p=0.70)$; there was a trend to lower UGI haemorrhage mortality in the OTSC group (0\% vs $4.7 \%, \mathrm{p}=0.09$ ).

Conclusions This is the first UK series to demonstrate that use of the OTSC for episodes of UGI haemorrhage requiring endoscopic therapy is associated with statistically significant lower rates of re-bleeding at 7 days. There is a trend towards reduced 30 day re-bleeding and UGI haemorrhage related mortality. Further studies are urgently required to assess whether this trend is statistically significant and to assess this exciting new modality for a condition where UK mortality has not been reduced for many years. 